\title{
DURACIÓN VOCÁLICA EN EL ESPAÑOL DE LA GRAN ÁREA METROPOLITANA DE COSTA RICA
}

\author{
Haakon S. Krohn
}

\section{(c) $(7)(\mathrm{F})$}

Doi: https://doi.org/10.15517/rfl.v45i1.36736

URL: https://revistas.ucr.ac.cr/index.php/filyling 



\title{
DURACIÓN VOCÁLICA EN EL ESPAÑOL DE LA GRAN ÁREA METROPOLITANA DE COSTA RICA
}

\author{
VOWEL DURATION IN THE SPANISH OF THE GREAT \\ METROPOLITAN AREA OF COSTA RICA
}

\author{
Haakon S. Krohn
}

\begin{abstract}
RESUMEN
En este artículo se describe un análisis acústico de la duración vocálica en palabras españolas leídas por estudiantes universitarios hispanohablantes de la Gran Área Metropolitana de Costa Rica. Los resultados revelan tres factores en el contexto lingüístico que aumentan la duración de las vocales en palabras bisilábicas. En primer lugar, las vocales en la primera sílaba son más largas cuando son tónicas que cuando son átonas. Asimismo, las vocales en la primera sílaba son más largas cuando la sílaba es abierta que cuando es cerrada. Por último, las vocales acentuadas en sílaba cerrada son más largas cuando esta sílaba es la segunda de la palabra que cuando es la primera.

Palabras clave: Español de Costa Rica; fonética acústica; vocales; duración; prosodia.
\end{abstract}

\begin{abstract}
This paper describes an acoustic analysis of the vowel duration in Spanish words read by Spanish speaking university students from the Great Metropolitan Area of Costa Rica. The results reveal three factors in the linguistic context that increase the vowel duration in disyllabic words. Firstly, vowels in the first syllable are longer when stressed than when unstressed. Moreover, vowels in the first syllable are longer when the syllable is open than when it is closed. Finally, stressed vowels in closed syllables are longer when they are in the second syllable of the word than in the first one. Key words: Costa Rican Spanish; acoustic phonetics; vowels; duration; prosody.
\end{abstract}

\section{Introducción}

El presente trabajo es una primera aproximación al estudio acústico de la duración vocálica en el español de Costa Rica. Como bien se sabe, esta característica no tiene valor fonológico en la variedad lingüística estudiada, pero cambia de acuerdo con una gran cantidad de factores, tanto globales como estructurales. El objetivo consiste en identificar factores en el contexto lingüístico que instiguen mayores duraciones de las vocales en palabras leídas por estudiantes universitarios provenientes de la Gran Área Metropolitana.

M. L. Haakon Stensrud Krohn. Universidad de Costa Rica. Profesor de la Escuela de Filología, Lingüística y Literatura. Costa Rica.

Correo electrónico: hkrohn@gmail.com

Recepción: 06- 02- 2018

Aceptación: 15- 04- 2018 
Los análisis de este fenómeno prosódico forman parte de la lingüística descriptiva. En lo que concierne al español, la duración vocálica ha sido estudiada acústicamente en muchas ocasiones, pero nunca se han tomado en consideración las variedades habladas en Costa Rica en este tipo de indagaciones. Los aportes más tempranos acerca de la duración vocálica en español son los de Navarro Tomás (1916; 1917). Posteriormente, destacan unos trabajos de Quilis $(1965 ; 1971)$ sobre el tema. En décadas más recientes, se han realizado numerosos estudios, gracias a las nuevas tecnologías que facilitan el proceso. Muchos de estos indagan en el español peninsular, por ejemplo, Monroy-Casas (1980), Marín (1994-1995), Cuenca (19961997) y Fernández y Martínez (2003). Por otro lado, Almeida (1986), Troya (2008-2009) y Dorta y Jorge (2015) analizan diversas variedades del español canario. Respecto al español americano, se pueden destacar los trabajos de Hammond y Resnick (1975) sobre el español cubano; Quilis y Esgueva (1983) y Chládková, Escudero y Boersma (2011) con informantes tanto de España como de Hispanoamérica; Vaquero y Guerra (1992) acerca del español de San Juan de Puerto Rico; Pereira y Soto (2011) enfocado en variedades diastráticas de Nuble en Chile; Mora, Rodríguez y Cavé (2011), sobre el español de Venezuela; y García (2014) enfocado en el español amazónico peruano.

\section{Selección de variables}

Con base en las obras mencionadas, se decidió considerar las siguientes cinco variables en el diseño de nuestro experimento; la primera tiene que ver con la naturaleza de la vocal en sí, mientras que las otras cuatro se vinculan con el contexto lingüístico:

- Duración intrínseca

- Consonante siguiente

- Condición acentuada/inacentuada

- Estructura silábica (abierta o cerrada)

- Posición en la palabra (primera o segunda sílaba)

En primer lugar, varios estudios (cf. Toivonen et al., 2014, p. 64) indican que, de manera universal, existe una correlación entre la altura de la vocal y la duración: las vocales altas tienden a ser más breves que las bajas. En consecuencia, se puede postular que cada vocal posee una duración intrínseca, relacionada con su altura. Este fenómeno también ha sido identificado en el español en diversas ocasiones, como se evidencia en Navarro Tomás (1916, 1917), Monroy-Casas (1980), Marín (1994-1995) y Troya (2008-2009), entre otros.

En segundo lugar, se incluyó la posibilidad de que la duración de la vocal sea influida por la consonante que le sigue, pues así lo afirman autores como Navarro Tomás (1916) y Almeida (1986). En particular, dichos lingüistas señalan que la vocal tiende a hacerse más larga ante consonantes sonoras. Sin embargo, otros autores, (por ejemplo, Marín, 1994-1995), no han llegado a la misma conclusión para las variedades estudiadas.

A su vez, la inclusión de la variable de la acentuación es evidente, ya que la duración es uno de los correlatos por medio de los cuales se manifiesta el acento léxico (junto con la frecuencia fundamental y la amplitud; Llisterri, et al., 2016, p. 1), y prácticamente todos los estudios que consideran dicha variable muestran mayores duraciones en las vocales tónicas que en las átonas. Asimismo, se consideró pertinente tomar en cuenta la dicotomía de sílaba abierta/cerrada, ya que este es un factor estudiado por varios autores (Navarro Tomás, 1916; Almeida, 1986; Marín, 1994-1995; Cuenca, 1996-1997; entre otros), de los cuales algunos 
concluyen que tiene relevancia significativa para la duración vocálica y otros que no. Por ejemplo, Marín (1994-1995) sí identifica mayor duración vocálica en sílabas abiertas, pero solo en posición prepausal. Almeida (1986) también encuentra diferencias, pero estas son mucho mayores en el estilo informal que en el formal (y no realiza ningún análisis de la significancia estadística de los resultados). Cuenca (1996), por su lado, no detecta disparidades estadísticamente significativas entre sílaba abierta y cerrada.

Para delimitar el estudio, no se tomó en consideración la variable del número de sílabas en la palabra; se trabajó solamente con palabras bisilábicas. Dada esta homogeneidad del corpus, queda natural incluir la variable de la posición en la palabra: primera o segunda sílaba. Finalmente, se decidió no tomar en cuenta vocales en posición final absoluta, puesto que, en ese entorno, la duración es muy cambiante y más difícil de medir con exactitud ${ }^{1}$.

\section{Método}

\subsection{Material}

El corpus utilizado en el experimento consiste en 75 palabras bisilábicas españolas, seleccionadas de tal modo que las cinco variables bajo estudio se representaran equilibradamente (con la excepción de 15 de las palabras, hecho que se explicará más adelante). En cada palabra se tomó en consideración solo una de las dos vocales, tanto en la elaboración del corpus como en el análisis de los resultados. Por eso, al referirnos en este apartado a la cantidad de palabras en la que aparezca determinado factor, se hace caso omiso de una de las dos vocales en cada palabra. Asimismo, para asegurar que las demás variables estuvieran equilibradas y no influyeran en el análisis de otra variable, no todas las palabras fueron tomadas en cuenta en todos los análisis. El corpus completo, con las vocales analizadas marcadas en negrita, se aprecia en el anexo 1 .

Es necesario especificar varios detalles acerca de la selección de las palabras. Primeramente, cada una de las cinco vocales aparece en quince palabras, en las que se presentan combinaciones de los cuatro factores contextuales relevantes para el estudio. Además, todas las vocales analizadas se ubican inmediatamente detrás de un fonema consonántico oclusivo ${ }^{2}$.

Cada una de las vocales analizadas va seguida por /n/, /s/ y / / en un tercio de los casos, respectivamente, por si la consonante siguiente tuviera alguna influencia en la duración vocálica. Se escogieron dichas consonantes por ser las únicas con las que se pudieron encontrar palabras que presentaran todas las otras combinaciones de variables requeridas para el estudio ${ }^{3}$.

También cabe mencionar que en solo 15 de las palabras (3 con cada vocal) la vocal relevante aparece en la última sílaba. Todas estas palabras son agudas y se tomaron en cuenta únicamente para los análisis de dos variables: la duración intrínseca y la posición en la palabra. Este último análisis es la razón por la que fue necesario incluir dichas palabras. Se excluyeron de las demás indagaciones debido al desequilibrio que habría sido causado por la inexistencia de palabras españolas graves con las cinco vocales seguidas por las consonantes /n/, /s/ y /r/ en la última sílaba. Por lo tanto, se puede decir que se trabajó con un corpus nuclear de 60 palabras, con las 15 palabras adicionales utilizadas solo para dos de los análisis.

En lo que respecta a la condición acentuada/inacentuada, la mitad de las vocales del corpus nuclear son de cada tipo. Lo mismo vale para la variable del tipo silábico abierto/ 
cerrado. Por último, en el análisis del factor de la posición en la palabra (primera o segunda sílaba) se contrastaron las 15 palabras en las que la vocal relevante aparece en la última sílaba con las 15 palabras graves que llevan la vocal relevante en una sílaba cerrada acentuada.

Lo descrito en este apartado implica que en ninguno de los análisis, excepto el de la duración intrínseca, la duración de cada una de las cinco vocales fue estudiada por separado. Por lo tanto, los resultados no muestran si las variables propuestas influyen de igual manera a todas las vocales. La decisión de dejar de lado este aspecto se debe al hecho de que se trata de una primera aproximación al tema de estudio; se requeriría un corpus sustancialmente mayor para obtener resultados significativos al respecto.

Cada palabra del corpus fue insertada en la oración "Digo la palabra ____,, con el objetivo de que todas fueran pronunciadas en el mismo contexto. Decidimos colocarlas al final de la oración para que no hubiera ninguna palabra posterior que corrompiera el análisis de la duración vocálica según la posición en la palabra. Finalmente, las 75 oraciones fueron imprimidas en tarjetas separadas.

\subsection{Participantes}

La muestra analizada proviene de grabaciones de audio de ocho estudiantes voluntarios (cuatro mujeres y cuatro hombres) de la Maestría Académica en Lingüística de la Universidad de Costa Rica. Todos tenían el español como lengua materna y procedían de la Gran Área Metropolitana de Costa Rica, donde habían residido toda su vida sin interrupciones significativas. Al momento de realizar el experimento, tenían una edad de entre 22 y 35 años. Por tanto, el grupo de participantes es relativamente homogéneo en cuanto a las variables de procedencia geográfica, edad y nivel de instrucción.

\subsection{Procedimiento}

Las grabaciones se realizaron con un participante a la vez, en el Edificio de la Facultad de Letras en la Ciudad Universitaria Rodrigo Facio de la Universidad de Costa Rica. Las 75 tarjetas con las oraciones se le fueron mostrando una por una, en orden aleatorio, a cada locutor. Antes de iniciar las sesiones, se les pidió que leyeran las oraciones con velocidad normal. Las sesiones completas fueron grabadas en Audacity (versión 2.1.3; Audacity Team, 2017) por medio de un micrófono JTS TX-8 con una respuesta de frecuencia de $50 \mathrm{~Hz}$ a 16 $\mathrm{kHz}$. Posteriormente, fueron guardadas en formato WAV.

\subsection{Análisis de los datos}

Se utilizó el software Praat (versión 6.0.30; Boersma y Weenink, 2017) para realizar los análisis de los datos. Con base en los oscilogramas y los espectrogramas, se calculó la duración, en milisegundos, de la vocal relevante en cada palabra. Estos valores fueron anotados en una hoja de cálculo en LibreOffice Calc (versión 5.4.1.2; LibreOffice Project, 2017).

Después de registrar la duración de todas las vocales bajo estudio, se empleó el software R (versión 3.4.1; R Core Team, 2017) para calcular los valores promedios según las distintas variables, así como la desviación estándar de cada uno de estos, con el fin de proporcionar una medida de dispersión. Además, utilizando el mismo programa, los resultados fueron sometidos a análisis de la varianza (ANOVA) para comprobar si las diferencias halladas eran estadísticamente significativas, con base en un nivel de significancia de 0,05. 


\section{Resultados}

En este apartado se presentan los resultados del estudio, según las variables consideradas. Cada tabla incluye cuatro columnas que contienen la siguiente información, respectivamente: valor de la variable, duración media ${ }^{4}$, desviación estándar (DE) y tamaño de muestra $(n)$.

\subsection{Duración intrínseca}

En el primer análisis, que indaga en la duración intrínseca de las vocales, se tomaron en cuenta todas las 15 instancias de cada vocal, lo cual (multiplicado por los 8 locutores) da un tamaño de muestra de 120. Los resultados se presentan en la tabla 1.

Como se puede observar, estos números concuerdan con la teoría de que la duración se correlaciona positivamente con la abertura vocálica: la vocal más abierta, /a/, es la que presenta la mayor duración media (92 ms), mientras que las más cerradas, /i/ y /u/, presentan la menor duración (84 ms). Sin embargo, como consecuencia de una desviación estándar de 23-24 ms en todos los casos, el ANOVA da un valor $p$ de 0,051 , lo cual quiere decir que, por un margen mínimo, ninguna de estas diferencias es estadísticamente significativa. Por lo tanto, los datos no son suficientes para concluir nada respecto a la duración intrínseca de las vocales, pero sí sugieren que es muy probable que exista una correlación entre la duración y la abertura vocálica.

Tabla 1. Duración media de las vocales según el fonema vocálico

\begin{tabular}{|c|c|c|c|}
\hline & $\begin{array}{c}\text { Duración } \\
\text { media } \\
(\mathbf{m s})\end{array}$ & DE (ms) & $\boldsymbol{n}$ \\
\hline$/ \mathrm{a} /$ & 92 & 23 & 120 \\
\hline$/ \mathrm{o} /$ & 88 & 23 & 120 \\
\hline$/ \mathrm{e} /$ & 87 & 24 & 120 \\
\hline$/ \mathrm{i} /$ & 84 & 24 & 120 \\
\hline$/ \mathrm{u} /$ & 84 & 24 & 120 \\
\hline
\end{tabular}

\subsection{Duración según la consonante siguiente}

El siguiente análisis, cuyos resultados se muestran en la tabla 2, toma en cuenta la consonante posvocálica como variable. Puesto que la / $/$ / tiende a ensordecerse a final de palabra en el español costarricense (Quesada y Vargas, 2010, p. 169), hecho evidenciado también en nuestras grabaciones, no se consideraron las vocales ubicadas en la segunda sílaba de la palabra, lo que da un tamaño de muestra de 160 para cada valor de la variable.

Los promedios de duración de las vocales, de entre 86 y $82 \mathrm{~ms}$, son muy similares ante las tres consonantes, lo cual sugiere que este factor no presenta mayor impacto. Además, la desviación estándar es relativamente alta (22-26 ms). Efectivamente, por medio del ANOVA se muestra que ninguna de estas diferencias es estadísticamente relevante $(p=0,361)$. 
Tabla 2. Duración media de las vocales según la consonante siguiente

\begin{tabular}{|c|c|c|c|}
\hline & $\begin{array}{c}\text { Duración } \\
\text { media } \\
(\mathbf{m s})\end{array}$ & DE (ms) & $\boldsymbol{n}$ \\
\hline$/ \mathrm{r} /$ & 86 & 26 & 160 \\
\hline$/ \mathrm{s} /$ & 85 & 23 & 160 \\
\hline$/ \mathrm{n} /$ & 82 & 22 & 160 \\
\hline
\end{tabular}

\subsection{Duración según la condición acentuada/inacentuada}

Respecto a la significancia de la condición acentuada/inacentuada, se tomaron en consideración todas las palabras en las que la vocal relevante está en la primera sílaba, al igual que en el análisis anterior. En este caso, el tamaño de la muestra es de 240 para cada valor de la variable. Los resultados de este análisis se exponen en la tabla 3.

Con una duración media de 99 ms, las vocales acentuadas son notablemente más largas que las inacentuadas, de $70 \mathrm{~ms}$. Esta diferencia sí es estadísticamente significativa, de acuerdo con los resultados del ANOVA $\left(p<2 \cdot 10^{-16}\right)$.

Tabla 3. Duración media de las vocales según la condición acentuada/inacentuada

\begin{tabular}{|c|c|c|c|}
\hline & $\begin{array}{c}\text { Duración } \\
\text { media } \\
(\mathbf{m s})\end{array}$ & DE (ms) & $\boldsymbol{n}$ \\
\hline Acentuada & 99 & 19 & 240 \\
\hline Inacentuada & 70 & 19 & 240 \\
\hline
\end{tabular}

\subsection{Duración según la estructura silábica}

La duración vocálica según la existencia de una consonante en la coda silábica es el factor estudiado en el cuarto análisis. Las sílabas abiertas presentan la estructura CV, mientras que las cerradas son del tipo CVC. También aquí, se tomaron en cuenta solamente las vocales en primera sílaba de la palabra.

Como se observa en la tabla 4, las vocales en sílaba abierta son más largas que las que se hallan en sílaba cerrada: $89 \mathrm{~ms}$ versus $79 \mathrm{~ms}$. La disparidad es estadísticamente significativa $\left(p=9,8 \cdot 10^{-6}\right)$.

Tabla 4. Duración media de las vocales según la estructura silábica (abierta o cerrada)

\begin{tabular}{|c|c|c|c|}
\hline & $\begin{array}{c}\text { Duración } \\
\text { media } \\
(\mathbf{m s})\end{array}$ & $\begin{array}{c}\text { DE } \\
(\mathbf{m s})\end{array}$ & $\boldsymbol{n}$ \\
\hline Abierta & 89 & 25 & 240 \\
\hline Cerrada & 79 & 21 & 240 \\
\hline
\end{tabular}




\subsection{Duración según la posición en la palabra}

En última instancia, se indagó en la diferencia de duración de las vocales en primera y segunda sílaba, respectivamente. En este caso, el tamaño de la muestra es de solo 120 para cada variable, por el hecho de que solo se consideraron vocales en sílabas acentuadas.

Los números en la tabla 5 ilustran que las vocales presentan una duración media de 99 ms en la segunda sílaba de la palabra, frente a $92 \mathrm{~ms}$ en la primera sílaba. La significación estadística de esta diferencia fue confirmada por el ANOVA $(p=0,0077)$.

Tabla 5. Duración media de las vocales según la posición en la palabra (primera o segunda sílaba)

\begin{tabular}{|l|c|c|c|}
\hline & $\begin{array}{c}\text { Duración } \\
\text { media } \\
(\mathbf{m s})\end{array}$ & $\begin{array}{c}\text { DE } \\
(\mathbf{m s})\end{array}$ & $\boldsymbol{n}$ \\
\hline Segunda sílaba & 99 & 22 & 120 \\
\hline Primera sílaba & 92 & 17 & 120 \\
\hline
\end{tabular}

\section{Discusión}

En primer lugar,el análisis de la duración intrínseca no arrojó resultados estadísticamente significativos, pero el valor $p$ apenas superó el nivel de significancia de 0,05 , por lo que es probable que en las vocales de la variedad lingüística estudiada sí se pueda encontrar una correlación positiva entre la duración y la abertura, lo cual apoyaría el postulado de que esta tendencia es universal.

En segundo lugar, no se pudo identificar ningún patrón que vinculara la duración vocálica con las características de la consonante siguiente. Empero, esto no descarta que en un estudio de un corpus más extenso de la misma variedad lingüística se revele algún efecto de dicha variable, sobre todo si se incluye una mayor variedad de consonantes posvocálicas.

En cuanto a las tres últimas variables -condición acentuada/inacentuada, estructura silábica y posición en la palabra- todas influyen significativamente en la duración vocálica en la variedad lingüística analizada. Estos hallazgos no se pueden cotejar directamente con los estudios de otras variedades del español, ya que las variables consideradas y las metodologías son distintas en cada caso. Las indagaciones más comparables con la presente, en lo que concierne a la selección de variables, son las del español peninsular de Marín (1994-1995) y Cuenca (1996) y la del español canario de Almeida (1986). En las tres se encontró que tanto la acentuación como la posición final propagan mayor duración vocálica. Sin embargo, como deja claro la reseña de los resultados de dichos estudios en el apartado 2, el efecto del factor de estructura silábica es más destacable en nuestro experimento.

\section{Conclusiones}

De los resultados se pueden extraer las siguientes conclusiones respecto a las palabras bisilábicas en la variedad lingüística estudiada:

- Las vocales en la primera sílaba son más largas cuando la sílaba es acentuada que cuando es inacentuada. 
- Las vocales en la primera sílaba son más largas cuando la sílaba es abierta que cuando es cerrada.

- Las vocales acentuadas en sílaba cerrada son más largas cuando esta sílaba es la segunda de la palabra que cuando es la primera.

En resumen, tanto la tonicidad, la estructura silábica abierta y la posición en la segunda (versus la primera) sílaba de la palabra son factores que parecen fomentar mayor duración vocálica.

Futuros estudios podrán, posiblemente, revelar otros factores relevantes, sobre todo si se basan en muestras más grandes o consideran variables diferentes, por ejemplo, la cantidad de sílabas en la palabra y la posición final absoluta versus otras posiciones. Asimismo, es importante enfatizar que los datos de nuestra indagación solo representan una variante diafásica específica: la lectura de oraciones aisladas. Por esta razón, es recomendable realizar análisis similares de otros registros, como el habla espontánea. En tal caso, sería imprescindible tomar en cuenta la velocidad de elocución, lo cual no se consideró necesario en el presente estudio, por el hecho de utilizar un corpus de palabras cortas.

Además, en este trabajo, las variables fueron analizadas de modo separado; no se tomó en cuenta la interacción de distintas variables. La variación individual de los hablantes tampoco fue considerada, para lo cual haría falta un corpus más grande.

\section{Notas}

1. Más que nada como consecuencia del fenómeno conocido como alargamiento final o prepausal (Fletcher, 2010, p. 540). Debido a las variaciones en el grado de alargamiento, la inclusión de vocales en posición final absoluta haría que la significancia estadística de los resultados disminuyera notablemente.

2. En algunas de las palabras, dicha consonante es sonora, debido a que no se logró encontrar una palabra con oclusiva sorda en esa posición. Por lo tanto, en ciertas ocasiones, la consonante fue pronunciada como aproximante o fricativa, por hallarse en un entorno intervocálico. Sin embargo, la mayoría de las consonantes sonoras intervocálicas fueron realizadas como oclusivas, posiblemente por el carácter cuidadoso del habla producida.

3. Principalmente porque pocas de las consonantes propias del sistema fonológico del español aparecen en posición final de sílaba.

4. Nótese que, dada la naturaleza del objeto de estudio, las duraciones no se pudieron medir con mayor exactitud que por milisegundos, de ahí que los valores de los promedios y de las desviaciones estándar tampoco incluyan decimales.

\section{Bibliografía}

Almeida, M. (1986). La cantidad vocálica en el español de Canarias: estudio acústico. Revista de filología de la Universidad de La Laguna, 5, 73-82.

Audacity Team. (2017). Audacity: Free Audio Editor and Recorder (versión 2.1.3) [software]. Recuperado de http://www.audacityteam.org

Boersma, P. y Weenink, D. (2017). Praat: Doing Phonetics by Computer (version 6.0.30) [software]. Ámsterdam: Department of Language and Literature, University of Amsterdam. Recuperado de http://www.praat.com

Chládková, K.; Escudero, P. y Boersma, P. (2011). Context-specific acoustic differences between Peruvian and Iberian Spanish vowels. Journal of Acoustical Society of America, 130 (1), 416-428. 
Cuenca, M. H. (1996). Análisis instrumental de la duración de las vocales en español. Philologia Hispalensis, 11 (1996-7), 295-307.

Dorta, J. y Jorge, C. (2015). Estudios prosódicos en Canarias: análisis de la duración en habla formal de El Hierro y Fuerteventura. Estudios de Fonética Experimental, 24, 11-33.

Fernández Planas, A. M. y Martínez Celdrán, E. (2003). El tono fundamental y la duración: Dos aspectos de la taxonomía prosódica en dos modalidades de habla (enunciativa e interrogativa) del español. Estudios de Fonética Experimental, 12, 165-200.

Fletcher, J. (2010). The prosody of speech: timing and rhythm. En W. J. Hardcastle, J. Laver y F. E. Gibbon (Eds.). The handbook of phonetic sciences. (pp. 523-602). West Sussex: Wiley-Blackwell.

García, M. (2014). Sobre la duración vocálica y la entonación en el español amazónico peruano. Lengua y Sociedad, 14 (2), 5-29.

Hammond, R. y Resnick, M. (1975). The status of quality and length in Spanish vowels. Jezikoslovlje, 13, 79-88.

LibreOffice Project (2017). LibreOffice Calc (versión 5.4.1.2) [software]. Recuperado de http:// www.libreoffice.org

Llisterri, J.; Machuca, M. J.; Ríos, A. y Schwab, S. (2016). La percepción del acento léxico en un contexto oracional. Loquens, 3, (2). doi: http://dx.doi.org/10.3989/loquens.2016.033

Marín Gálvez, R. (1994-1995). La duración vocálica en español. Estudios de Lingüística Universidad de Alicante, 10, 213-226.

Monroy-Casas, R. (1980). Aspectos fonéticos de las vocales españolas. LibrosEnRed.

Mora, E.; Rodríguez, M. y Cavé, C. (2007). La duración de la vocal simple y de las homólogas en el español venezolano: el caso de pares mínimos. Lenguaje, 35, (1), 11-27.

Navarro Tomás, T. (1916). Cantidad de las vocales acentuadas. Revista de Filología Española, 3, 387-408.

Navarro Tomás, T. (1917). Cantidad de las vocales inacentuadas. Revista de Filología Española, 4, 371-388.

Pereira, D. I. y Soto-Barba, J. (2011). Duración absoluta de las vocales del español urbano y rural de la provincia de Nuble. Boletín de Filología, 46, (1), 153-161.

Quesada Pacheco, M. A. y Vargas Vargas, L. (2010). Rasgos fonéticos del español de Costa Rica. En M. A. Quesada Pachecho (Ed.). El español hablado en América Central: nivel fonético. España: Editorial Iberoamericana / Vervuert.

Quilis, A. (1965). Phonologie de la quantité en espagnol. Phonetica, 13, 82-85.

Quilis, A. (1971). Caracterización fonética del acento en español. Travaux de Linguistique et de Littérature, 9, 53-72.

Quilis, A. y Esgueva, M. (1983). Realización de los fonemas vocálicos españoles en posición fonética normal. En M. Esgueva y M. Cantero (Eds.). Estudios de fonética, vol. 1. (pp. 159-252). Madrid: Centro Superior de Investigaciones Científicas. 
R Core Team. (2017). R: A Language and Environment for Statistical Computing (version 3.4.1) [software]. Recuperado de http://www.r-project.org

Toivonen, I.; Blumenfeld, L.; Gormley, A.; Hoiting, L.; Logan, J.; Ramlakhan, N. y Stone, A. (2014). Vowel height and duration. En U. Steindl, et al. (Eds.). (pp. 64-71). Proceedings of the 32nd West Coast Conference on Formal Linguistics.

Troya Déniz, M. (2008-2009). La duración de las vocales tónicas en la norma culta de Las Palmas de Gran Canaria. Philologica Canariensia, 14-15, 297-312.

Vaquero, M. T. y Guerra, L. (1992). Fonemas vocálicos de Puerto Rico (análisis acústico realizado con los materiales grabados para el estudio de la norma culta de San Juan). Revista de Filología Española, 72, (3-4), 555-582.

\section{Anexo 1}

Palabras incluidas en el corpus, con las vocales analizadas en negrita y los lindes silábicos representados por medio de guiones. $\mathrm{A}=$ abierta; $\mathrm{C}=$ cerrada .

\begin{tabular}{|c|c|c|c|c|c|c|c|}
\hline Acentuada & $\begin{array}{l}\text { Estructura } \\
\text { silábica }\end{array}$ & Sílaba & /a/ & /e/ & /i/ & $/ \mathrm{u} /$ & $/ 0 /$ \\
\hline \multirow{3}{*}{+} & \multirow{3}{*}{ A } & \multirow{3}{*}{$1^{\mathrm{a}}$} & ca-na & pe-na & pi-no & cu-na & to-no \\
\hline & & & ca-sa & pe-so & pi-so & pu-so & co-sa \\
\hline & & & ca-ro & pe-ra & ti-ro & pu-ro & to-ro \\
\hline \multirow{3}{*}{+} & \multirow{3}{*}{$\mathrm{C}$} & \multirow{3}{*}{$1^{\mathrm{a}}$} & can-ta & ten-so & pin-to & pun-to & ton-to \\
\hline & & & pas-ta & pes-te & pis-ta & gus-to & pos-te \\
\hline & & & par-te & ter-co & vir-go & pur-ga & tor-ta \\
\hline \multirow{3}{*}{-} & \multirow{3}{*}{ A } & \multirow{3}{*}{$1^{\mathrm{a}}$} & ga-nar & te-ner & pi-nar & pu-nir & do-nar \\
\hline & & & ca-sar & pe-sar & pi-sar & bu-zón & co-ser \\
\hline & & & pa-rar & que-rer & ti-rar & cu-rar & co-ral \\
\hline \multirow{3}{*}{-} & \multirow{3}{*}{$\mathrm{C}$} & \multirow{3}{*}{$1^{\mathrm{a}}$} & can-tar & pen-sar & pin-tar & pun-tal & bon-dad \\
\hline & & & cas-tor & ves-tir & pis-tón & bus-car & cos-tar \\
\hline & & & car-gar & ter-cer & bir-lar & cur-sar & cor-tar \\
\hline \multirow{3}{*}{+} & \multirow{3}{*}{$\mathrm{C}$} & \multirow{3}{*}{$2^{\mathrm{a}}$} & es-tán & re-tén & bo-tín & a-tún & ra-tón \\
\hline & & & ca-paz & mar-qués & sen-tís & cham-pús & pre-coz \\
\hline & & & ma-tar & me-ter & ba-tir & as-tur & mo-tor \\
\hline
\end{tabular}

